\title{
Coherence Versus Elicitability in Measures of Market Risk
}

\author{
James Ming Chen
}

Published online: 26 July 2014

(C) International Atlantic Economic Society 2014

JEL Categories F30 International Finance · F39 Global Financial Markets · G15 International Financial Markets · L50 Regulation and Industrial Policy

The second and third accords of the Basel Committee on Banking Regulation prescribe distinct measures of market risk. Basel II has embraced value-at-risk (VaR) analysis, while Basel III has proposed replacing VaR with expected shortfall. These risk measures suffer from mutually irreconcilable flaws. VaR fails to satisfy the conditions required of "coherent" risk measures. Conversely, expected shortfall fails the mathematical requirements for "elicitability." Mathematical limitations force a choice between theoretically sound aggregation of risks and reliable backtesting of forecasts.

$\mathrm{VaR}$ for confidence level $\in$ is the quantile that solves the following equation [Danielsson and Zigrand, Journal of Banking of Finance, 2006]:

$$
\epsilon=\int_{-\infty}^{-V a R} f(x) d x
$$

VaR does not qualify as a mathematically "coherent" risk measure. A risk measure is coherent if and only if it satisfies all four of these conditions: translation invariance, homogeneity, monotonicity, and subadditivity [Artzner et al., Mathematical Finance, 1999]:

1. Translation invariance: Adding constant return $c$ to total portfolio return reduces risk by $c$.

2. Homogeneity: Increasing any portfolio by a positive factor $\lambda$ requires a corresponding increase in regulatory capital.

J. M. Chen $(\bowtie)$

Justin Smith Morrill Chair in Law, Michigan State University College of Law, 648 North Shaw Lane, East Lansing, MI 48824-1300, USA

e-mail: chenjame@law.msu.edu 
3. Monotonicity: If one portfolio is first-order stochastically dominant in that it offers higher returns in every state, then the risk associated with that portfolio cannot be higher than that of any other.

4. Subadditivity: The risk associated with two combined subportfolios cannot exceed the total risk associated with each subportfolio.

Translation invariance, homogeneity, and monotonicity are not difficult to satisfy. Subadditivity suggests that a combined portfolio cannot be riskier than its components standing apart. Aggregation should lower risk through diversification. But VaR fails to satisfy subadditivity. VaR levels, calculated for separate portfolios, provide incoherent predictions of risk. Aggregating VaR 1 for different portfolio components can overstate the risk of the entire portfolio. VaR may also understate aggregated risks.

Basel III has proposed replacing VaR with expected shortfall. The expected shortfall for any continuous loss function may be expressed as conditional VaR or the tail conditional expectation - the expected loss conditional on the loss lying beyond the VaR quantile $\alpha$ [Ziegel, Coherence and Elicitability, 2013 (http://arxiv.org/pdf/1303. 1690v2)]:

$$
E S_{\alpha}(L)=\frac{1}{\alpha} \int_{0}^{\alpha} \operatorname{VaR}_{\tau}(L) d \tau
$$

Although expected shortfall, as the sum of all losses exceeding confidence interval $\alpha$, is a transformation of VaR, only expected shortfall is subadditive and coherent [Hull, Risk Management and Financial Institutions, 2012 (3rd edition)].

Though coherent, expected shortfall is not an ideal risk measure. Expected shortfall cannot be backtested against historical benchmarks. Ease of backtesting is VaR's primary advantage. The VaR quantile not only identifies the frequency of significant losses, but also triggers regulatory intervention.

Expected shortfall measures all risks in the tail of a distribution, including theoretically possible losses that are not observed during backtesting. Mathematically, expected shortfall is not elicitable [Gneiting, Journal of the American Statistical Association, 2011]. Indeed, nearly the entire class of subadditive and coherent spectral risk measures, of which expected shortfall is a special case [Acerbi and Tasche, Journal of Banking of Finance, 2002], is not elicitable.

By contrast, VaR, like any other quantile-based measure, is elicitable. An elicitable risk measure can be subjected to a consistent scoring function that forecasts future losses. The nonelicitability of expected shortfall creates a direct conflict with VaR. Expected shortfall addresses VaR's incoherence. But expected shortfall in turn eludes backtesting. Expected shortfall thus suffers from an intractable defect of its own.

Mathematics delivers an elegant denouement. Exactly one spectral risk measure is both coherent and elicitable. Only negative expected value satisfies both conditions [Ziegel, at 8]. To return to that measure of risk would, implausibly and imprudently, commit regulators to measuring risk according to expected return on the trading book as a whole, with no consideration of volatility, beta, or some other gauge of the dispersion of trading outcomes. The choice between VaR and expected shortfall therefore forces a choice between coherence and elicitability. 